Clegg, dem Vorsitzenden der Liberal Democrats, fünf Tage nach der Wahl, sich zu entscheiden. Als dieser weiter lavieren wollte, reagierte Brown mit seinem sofortigen Rücktritt.

Das Ende von New Labour hatte mehr mit dem Scheitern eines politischen Projekts zu tun, das von seinen Kritikern als Thatcherismus mit menschlicherem Antlitz gesehen wurde - ein Projekt, das auch große Diskussionen über Manipulationen der Öffentlichkeit und die Macht der Medien provozierte und Fragen aufwarf nach dem Sinn der „special relationship“ mit den USA, die keineswegs mehr als Allianz auf Augenhöhe wahrgenommen wurde. Liest man die drei Bände, so sind sich die Autoren in einem einig: New Labour war zwar am Ende - aber dafür, dass der Fall dieser Idee so tief ausfiel und die Labour Party die Wahlen von 2010 verlor, gibt es einen Schuldigen: Gordon Brown. An seiner Intelligenz und Gradlinigkeit werden von keinem der Autoren die geringsten Zweifel geäußert. Er erscheint mehr als tragische Figur. In den Worten eines britischen Politikwissenschaftlers: aus dem finanzpolitischen „Stalin“ wurde in der Öffentlichkeit Mr Bean. ${ }^{3}$

Roland Sturm

3 Mit „From Stalin to Mr Bean“ ist ein Kapitel des Aufsatzes von Nicholas Allen überschrieben; ders., Labour's Third Term: A Tale of Two Prime Ministers, in: ders. / John Bartle (Hrsg.), Britain at the Polls 2010, London 2011, S. 1 - 36, S. 8.

\title{
Rechtsextremismusforschung: einseitige Argumentation, verpasste Aufklärung
}

Forum für kritische Rechtsextremismusforschung (Hrsg.): Ordnung. Macht. Extremismus. Effekte und Alternativen des Extremismus-Modells, VS Verlag für Sozialwissenschaften, Wiesbaden 2011, 376 Seiten, $€ 29,95$.

Weiß, Volker: Deutschlands Neue Rechte. Angriff der Eliten - Von Spengler bis Sarrazin, Verlag Ferdinand Schöningh, Paderborn 2011, 141 Seiten, € 16,90.

Extremismus taucht als Bedrohung der Demokratie und ihrer Werte in vielen Facetten auf. Gerade in Deutschland ist das Thema durch die „Schatten der Vergangenheit“ äußerst sensibel. Doch nicht nur in der Geschichte, sondern auch in der Gegenwart steht der demokratische Verfassungsstaat vor ständigen Herausforderungen, die seine Feinde heraufbeschwören. Momentan entfacht das Bekanntwerden eines Rechtsterrorismus eine um sich greifende Debatte über das offenkundige Versagen der Sicherheitsbehörden auch im Bundestag. Ein Untersuchungsausschuss soll die Vorgänge erhellen. Den Sozialwissenschaften kommt damit eine gesellschaftspolitische Herausforderung zu, die über die akademische Welt hinausgeht. Ein viel beachtetes Konzept ist dabei die normative Extremismustheorie, die die beiden deutschen Politikwissenschaftler Uwe Backes und Eckhard Jesse mit unzähligen Publikationen bis heute, unter anderem mit dem Jahrbuch Extremismus \& Demokratie, geprägt haben. ${ }^{1}$

1 Vgl. Uwe Backes / Eckhard Jesse, Demokratie und Extremismus. Anmerkungen zu einem antithetischen Begriffspaar, in: APuZ, B 44/1983, S. 3 - 18. 
Bereits 1983 sprachen Jesse und Backes von Demokratie und Extremismus als einem antithetischen Begriffspaar. Das normative Extremismuskonzept begreift sich als „eine Anwendung des Totalitarismus- und Autoritarismuskonzepts auf diejenigen Kräfte innerhalb des demokratischen Verfassungsstaates, die diesem offen oder verdeckt den Kampf angesagt haben"2. Damit ist er aber entgegen dem universellen Anspruch auf die Bundesrepublik bezogen, da das Extremismuskonzept ganz wesentlich auf die grundgesetzlich verankerte streitbare oder wehrhafte Demokratie und damit auf deutsche Spezifika abstellt. Eckhard Jesse argumentiert unmissverständlich, fast gebetsmühlenhaft: „Wer das Extremismuskonzept in Frage stellt, negiert damit die Konzeption der streitbaren Demokratie, wie sie in der Bundesrepublik als Reaktion auf die leidvolle Vergangenheit gilt. "3 Immer wieder schlägt Backes und Jesse auch durch ihren exponierten, mitunter pointierten Vergleich (vor allem durch Jesse) zwischen Rechts- und Linksextremismus ein rauer Gegenwind entgegen. So sieht Jesse in zwei Parteien, der auch im medialen Diskurs etablierten Linken und der medial ausgegrenzten, aggressiv-kämpferischen NPD einen Extremismus in seiner weichen beziehungsweise harten Ausprägung. ${ }^{4}$ Für die Linke bedeutet das angesichts dessen, dass Extremismus und Demokratie wie „Feuer und Wasser"5 zueinander stünden, eine Provokation. Auch sonst bemüht sich Jesse aus der Perspektive der streitbaren Demokratie darum, den Linksextremismus als Demokratiegefahr ernst zu nehmen und den Rechtsextremismus gerade im parteiförmigen Spektrum nicht zu dramatisieren.

Nun übt ein Sammelband des Forums für kritische Extremismusforschung, ein Netzwerk an den Universitäten Leipzig und Frankfurt am Main, Fundamentalkritik an diesem Konzept, verbunden mit dem Anspruch einer grundsätzlichen Neuorientierung. Jörn Hüttmann plädiert dafür, begrifflich extreme Rechte statt Rechtsextremismus zu verwenden, da auch „in Teilen der ,Mitte der Gesellschaft" rechte Einstellungen verbreitet [seien]“ (S. 343). Eine Auffächerung des Extremismusbegriffs produziere lediglich „ein hegemoniales Projekt“ (S. 118), keinen wissenschaftlichen Erkenntnisgewinn. Dementsprechend lehnen die Autoren das Hufeisenmodell der normativen Extremismustheorie mit polemischen, unsachlichen Verweisen auf die Herren „Doktor Backes und Professor Doktor Jesse“ (sic!; S. 48) ${ }^{6}$ ab, wonach sich Rechts und Links an den Rändern berührten. Deren in der Tat vorhandene Nähe zu Mitarbeitern des Verfassungsschutzes, die sich auch durch die Anhängerschaft des Konzepts der streitbaren Demokratie ergibt, mache sie ohnehin verdächtig. Ein Beitrag von Matthias Falter trägt den bezeichnenden Titel „Critical Thinking beyond Hufeisen“, worin er den „postnationalsozialistischen Gesellschaften“ eine Abkehr von Erinnerung unterstellt. Gar ins

2 Eckhard Jesse, „Extremistische Parteien“: Worin besteht der Erkenntnisgewinn?, in: APuZ, B 47/2008, S. 7 - 10, S. 8.

3 Ebenda, S. 8 ff.

4 Vgl. Eckhard Jesse, Die NPD und die Linke. Ein Vergleich zwischen einer harten und weichen Variante des Extremismus, in: Uwe Backes / ders. (Hrsg.), Jahrbuch Extremismus \& Demokratie 2009, Baden-Baden 2010, S. 13 - 31.

5 Eckhard Jesse, Formen des politischen Extremismus, in: Bundesministerium des Innern (Hrsg.), Extremismus in Deutschland. Erscheinungsformen und aktuelle Bestandsaufnahme, Berlin 2004, S. $7-24$.

6 Hier käme auch dem Verlag eine wichtige Funktion zu. Offenbar werden einige sozialwissenschaftliche Publikationen in Großverlagen nicht mehr ordentlich, also wenn überhaupt schlampig redigiert. 
Lächerliche zieht Tobias Prüwer den Versuch von Uwe Backes ${ }^{7}$, eine Begriffsgeschichte des Extremismus von Aristoteles bis zum demokratischen Verfassungsstaat der Bundesrepublik zu ziehen und somit das Konzept zu stärken. Dies sei "geschichtslos" und „historisch blind“ (S. 61). Ganz unbegründet ist der Einwand aber nicht. So übersieht Backes geflissentlich, dass die damalige Extremismuserwähnung durch Aristoteles nicht im Zusammenhang mit dem Verständnis moderner demokratischer Verfassungsstaaten steht, das ja als entscheidende Projektionsfläche grundlegend für die Definition ist.

Der schon lange konstatierte Begriffswirrwarr bei der Beschreibung dieses Phänomens wird ebenso bemängelt. Auch der Populismusbegriff blende aus, dass die diskursiven Wurzeln des Extremismus in der gesellschaftlichen Mitte zu suchen seien: „Das eigentliche Thema des Extremen ist nicht der politische Rand, sondern der Mainstream, [...], wenn es zum Beispiel darum geht, Leitkulturen essentialistisch den Minoritäten entgegenzuhalten.“ (S. 366) Als Rechtfertigung dieser gewagten These werden vereinzelte Beispiele schlagwortartig herangezogen: die durch Geert Wilders befeuerte Islamophobie in den Niederlanden, die Sarrazin-Debatte sowie das Erstarken der ungarischen Jobbikpartei. An Kritik gegen staatliche Behörden wird dabei nicht gespart. So erfolge die Erfassung politisch motivierter Kriminalität häufig auf der linken Seite, obwohl die dort häufig georteten Sachbeschädigungen gar nicht politisch motiviert seien. In der Tat lassen sich, ein generelles Problem, Gewaltstraftaten nicht immer kontextualisieren. Aber auch im Rechtsextremismus steht medial geschürte Hysterie vielfach vor sachlicher Aufklärung, wie etwa der bis heute ungeklärte „Fall Mannichl“8 zeigt. Obwohl es viele Fragezeichen gab und gibt, beschloss die bayerische Staatsregierung ein umfassendes Maßnahmenpaket gegen Rechtsextremismus. Dafür steht auch der im Band thematisierte „Fall Mügeln“, eine Art von „moral panics“ (S. 206). Mitunter bedenkenswerte Aspekte im wenig konzisen Band gehen aber durch die unsachliche Herangehensweise und zuweilen akademisierte Sprache unter.

Auch Volker Weißsieht eine Neue Rechte aus der Mitte der Gesellschaft entspringen - als spezifisch deutsche Tradition. Die von ihm konstruierte Ahnenreihe ist lang und rechtfertigt den Begriff der Neuen Rechten nicht, da er die "Geistesströmung“ schon Ende des 19. Jahrhunderts ansetzt: Sie reicht von Oswald Spengler über Botho Strauß bis hin zu Thilo Sarrazin. Auch der Philosoph Peter Sloterdijk fällt darunter. Die „Brandstifter“ teilten „die Beschwörung des Untergangs der eigenen Kultur, meist in Verbindung mit demographischen Berechnungen und dem Ruf nach einer starken Elite“, also „apokalyptische Visionen“ (S. 11). Der Autor sieht damit eine intellektualisierte Strömung, die über biologische, teils sozialdarwinistische Annahmen die Legitimation einer generell höher begabten Menschengruppe herleitet. Allerdings wirkt es wenig schlüssig, über deutsche Systembrüche hinweg einen scheinbar konstanten elitären Dünkel abzuleiten, zumal die Autoren größtenteils gar nicht zueinander in Beziehung stehen. Der Untertitel spricht aber von einem „Angriff der Eliten“, wohl auf das gemeine Volk. Das mag für Sarrazins sensationellen Erfolg, das meistverkaufte politische Buch in der Bundesrepublik Deutschland, mit Fug und Recht gelten,

7 Vgl. Uwe Backes, Politische Extreme. Eine Wort- und Begriffsgeschichte, Göttingen 2006. Nun auch in englischer Sprache: Political Extremes. A Conceptual History From Antiquity to the Present, New York 2011.

8 Alois Mannichl, damals Leiter der Polizeidirektion der Stadt Passau, wurde bundesweit bekannt, als am 13. Dezember 2008 auf ihn vor seiner Haustür im Privathaus offenbar ein Messerangriff verübt wurde. 
doch schwerlich für das Studienzentrum Weikersheim, das keine Verkaufsschlager produziert und noch nie produziert hat. Doch auch Sarrazin ist bei weitem nicht so mächtig, „die politischen und geschäftlichen Interessen des Springer-Verlags und des Bertelsmann-Konzerns hinter sich [zu wissen]“ (S. 129). Immerhin ist die Studie von Weißflüssig geschrieben, ganz im Gegensatz zum besprochenen Sammelband.

Die beiden Publikationen machen deutlich, wie politisch aufgeladen die Debatte um den Rechtsextremismus in Deutschland ist. So fordert die Politik immer wieder reflexhaft in der deutschen „Betroffenheitsdemokratie“ das Verbot der NPD, ohne eine langfristig angelegte Strategie zu verfolgen. Der letzte Meinungsstreit ist hier nicht ausgetragen, zumal nach den lange unerkannt gebliebenen rechtsterroristischen Vorfällen. Eine neue Debatte um Befugnisse und Grenzen der staatlichen Observation, auch um Effektivität begleitet die nächste Zukunft deutscher Politik. Wenn aber Polemik „zum roten Faden“wird, wird die Ernsthaftigkeit und Bedeutung des Themas untergraben und eine sachliche Auseinandersetzung erschwert. Das Thema hat nicht nur in den Sozialwissenschaften und der praktischen Politik, sondern auch in der politischen und schulischen Bildung eine gewichtige Rolle zu spielen. Rechtsextremismus ist in erster Linie ein gesellschaftliches Phänomen. Wer bei dieser Vielschichtigkeit einseitig argumentiert, verwirkt diese Aufklärungsfunktion.

Florian Hartleb

\section{Extremismus in der EU: Hilfreiches Handbuch für Erstsemester wie Experten}

Jesse, Eckhard und Tom Thieme (Hrsg.): Extremismus in den EU-Staaten, VS Verlag für Sozialwissenschaften, Wiesbaden 2011, 505 Seiten, € 39,95.

Zu Beginn dieses Jahres haben in Ungarn Zehntausende gegen die von der Regierung unter Viktor Orbán beschlossenen Verfassungsänderungen demonstriert, die neben einer umfassenden Kompetenzerweiterung für den Ministerpräsidenten und einer Zensur der Presse unter anderem vorsehen, den Zusatz „Republik“ aus der offiziellen Staatsbezeichnung Ungarns zu tilgen. Dieses aktuelle Beispiel zeigt, dass demokratische Grundprinzipien in der Europäischen Union noch immer nicht als unangefochten gelten können. Es wird deutlich, wie vorschnell die berühmte These des US-amerikanischen Politikwissenschaftlers Francis Fukuyama gewesen ist, derzufolge die liberal-demokratische Idee siegreich aus dem Wettstreit der konkurrierenden Ideologien des 20. Jahrhunderts hervorgegangen ist. Obwohl Fukuyama damit den euphorischen intellektuellen Zeitgeist der Epochenwende 1989/91 auf den Punkt gebracht hat, sehen sich Demokratie und Verfassungsstaat auch heute noch mit vielfältigen Herausforderungen durch extremistische Bestrebungen konfrontiert. Das gilt auch für die EU, deren jüngste Entwicklung nicht zu Unrecht als Erfolgsgeschichte gefeiert wird, da sie dem Kontinent ein zuvor nie gekanntes Maß an Sicherheit und materiellem Wohlstand bescherte. Extremistisch-antiliberale Kräfte und Strömungen haben ihren historischen Zenit allerdings noch nicht überschritten. Sie erfordern nach wie vor die begleitende wissenschaftliche Beobachtung, Kommentierung und Einordnung.

Vor diesem Hintergrund beschäftigt sich der vom Chemnitzer Politologen Eckhard Jesse und seinem Mitarbeiter Tom Thieme besorgte Sammelband mit extremistischen Tendenzen 